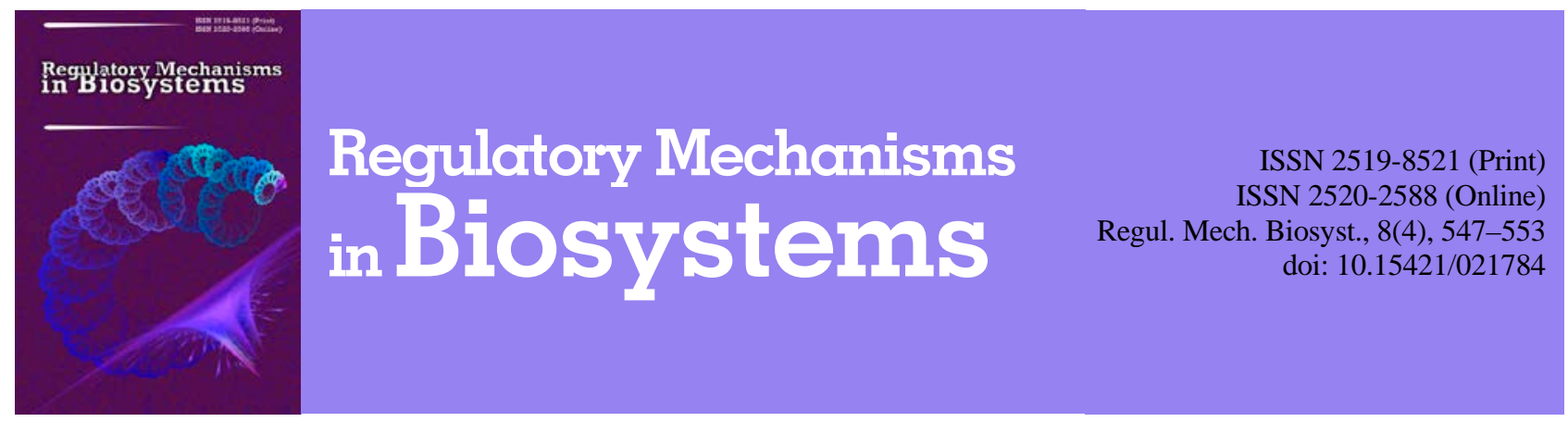

\title{
Improving the quality of life of patients with stable angina pectoris using combined therapy
}

\author{
K. O. Zolotarova \\ Odessa National Medical University, Odessa, Ukraine
}

Article info

Received 28.09.2017

Received in revised form 20.10.2017

Accepted 25.10.2017

Odessa National Medical University, Valikhovski prov., 2, Odessa, 65000, Ukraine. Tel. +38-048-723-42-49. E-mail:sugar.fm@gmail.com
Zolotarova, K. O. (2017). Improving the quality of life of patients with stable angina pectoris using combined therapy. Regulatory Mechanisms in Biosystems, 8(4), 547-553. doi:10.15421/021784

Treatment of chronic ischemic heart disease includes the use of non-drug methods (anti-hypodynamia, smoking cessation, etc.), as well as drug therapy, the main tasks of which are to reduce the risk of complications such as stroke, myocardial infarction, sudden cardiac death, and to gain an increase in life expectancy. An important aspect of treatment is also a decrease in the frequency and intensity of angina attacks, the need for short-acting nitrates, to gain an increase in physical activity, that is, an overall, improvement in the quality of life of patients. The article presents the results of studying the quality of life indicators of patients with stable angina pectoris with the use of omega-3 polyunsaturated fatty acids and magnetotherapy. 85 patients with stable angina pectoris were examined, divided into three groups according to the method of therapy. 28 patients of the I (control) group received a drug complex corresponding to the national protocol for the treatment of patients with angina pectoris: nitrates, beta-blockers, ACE inhibitors, antiplatelet drugs, statins (atorvastatin at a daily dose of $20 \mathrm{mg}$ ). 29 patients of the II group, together with protocol therapy, received the drug omega-3 polyunsaturated fatty acids in a daily dosage of $2000 \mathrm{mg}$, divided into 2 doses. In connection with the known hypolipidemic action of omega-3 PUFA, the daily dose of atorvastatin in this and the next group was reduced to $10 \mathrm{mg}$. Group III consisted of 28 patients who were prescribed omega-3 polyunsaturated fatty acids and a course of magnetotherapy according to a special method against the background of the protocol medication complex. It was revealed that, initially in patients with angina, the quality of life indicators according to the Seattle questionnaire and the visual analogue scale were reduced. It was shown that with the use of the protocol medication complex, all the parameters of the quality of life improved reliably, with a significant reduction in the angina pain scales and less significant improvement on the scales of physical activity, assessments of health and the perception of the disease . Upon adherence to the therapy of omega-3 polyunsaturated fatty acids, there was a positive but very weak tendency to improve the quality of life indicators, which did not differ significantly from the control. The use of combined therapy with simultaneous inclusion of omega-3 polyunsaturated fatty acids and magnetotherapy led to significant and reliable improvement in the quality of life indicators, not only on the scales reflecting the pain component, but also on the indicators that characterize the psychological contribution to the quality of life (treatment satisfaction and quality of life (disease perception)). The significant improvement in the quality of life in patients receiving magnetotherapy in contrast to the absence of such influence in patients receiving omega-3 polyunsaturated fatty acids without magnetotherapy was due to the strong antianginal effect of MT, its sedative and emotionally relaxing action, and the ability to enhance the effects of medications.

Keywords: ischemic heart disease; Seattle Angina questionnaire; visual analogue scale; omega-3 polyunsaturated fatty acids; magnetotherapy

\section{Улучшение качества жизни больных стабильной стенокардией напряжения при применении комбинированной терапии}

\author{
К. О. Золотарева \\ Одесский наџиональный медищинский университет, Одесса, Украина
}

Представлены результаты изучения показателей качества жизни больных стабильной стенокардией напряжения при использовании омега-3 полиненасыщенных жирных кислот и магнитотерапии. Обследовано 85 больных стабильной стенокардией напряжения I-III функциональных классов, разделенных на три группы по способу терапии. 28 больных контрольной группы для лечения получали протокольный медикаментозный комплекс для больных стенокардией, включающий нитраты, $\beta$-блокаторы, ингибиторы АПФ, антитромбоцитарные препараты, статины. 29 больным II группы совместно с протокольным комплексом назначался препарат омега-3 полиненасыщенных жирных кислот (витрум кардио омега-3) в суточной дозировке 2000 мг. 28 больным III группы на фоне протокольного медикаментозного комплекса назначались омега-3 полиненасыщенные жирные кислоты и курс магнитотерапии по специальной методике. 
Исходно качество жизни больных значительно снижено, согласно показателям Сиэттлского опросника для больных стенокардией, а также показателям визуальной аналоговой шкалы. При применении протокольного медикаментозного комплекса отмечалось достоверное улучшение показателей качества жизни, однако, в основном за счет выраженного снижения количества ангинозных приступов, в то время как показатели ограничения физической активности, оценки самочувствия и восприятия болезни увеличились также достоверно, но менее выраженно. При добавлении к протокольному комплексу омега-3 полиненасыщенных жирных кислот по всем показателям качества жизни значительного и достоверного улучшения не отмечалось, хотя общая тенденция была положительной. При совместном использовании $́$-3 ПНЖК и магнитотерапии эффект воздействия на все показатели качества жизни был максимальным, причем не только по шкалам приступов стенокардии, но также и по показателям физической активности, удовлетворенности лечением, оценки самочувствия и, в итоге, по общему качеству жизни. Данный эффект обусловлен наличием помимо антиангинального эффекта магнитотерапии, ее седативным и снимающим эмоциональное напряжение действием.

Ключевые слова: ишемическая болезнь сердца; Сиэттлский опросник; визуальная аналоговая шкала; омега-3 полиненасыщенные жирные кислоты; магнитотерапия

\section{Введение}

Лечение хронической ишемической болезни сердца (ИБС) включает в себя использование немедикаментозных методов (борьба с гиподинамией, отказ от курения и т. д.), а также медикаментозную терапию, основными задачами которой является снижение риска развития осложнений, таких как инсульт, инфаркт миокарда, внезапная сердечная смерть, а также увеличение продолжительности жизни. Важным аспектом лечения является также использование методов, направленных на уменьшение частоты и интенсивности ангинозных приступов, потребности в приеме нитратов, увеличение физической активности, то есть практически всех составляющих, входящих в понятие «качество жизни» больного стенокардией.

Термин качество жизни (КЖ) охарактеризован Всемирной организацией здравоохранения как «индивидуальное восприятие своей позиции в жизни в контексте с культурной средой и системой ценностей, в которой проживает индивид, и в соотношении с его целями, ожиданиями, стандартами и воззрениями». Начало изучения качества жизни современной медициной приходится на вторую половину XX века, когда стало очевидно, что в процессе лечения важно добиться не только редукции болезненных симптомов, но и улучшения качества жизни и социального функционирования больного (Zholdasbekova et al., 2016). В последние десятилетия наблюдается повышение интереса к исследованиям КЖ, а также возрастание роли этих исследований, что отражается в динамике числа публикаций по данной проблеме (Osipov et al., 2011).

Основными инструментами изучения качества жизни являются всевозможные анкеты, опросники, визуальные аналоговые шкалы, поскольку именно они позволяют оценить субъективное состояние больного, его отношение к своей болезни, степень влияния этой болезни на его повседневную жизнь. Методики, используемые в кардиологической практике, подразделяются на общие, то есть применяющиеся независимо от нозологии, а также специальные - разработанные с учетом конкретного заболевания или ведущего синдрома. Из общих методик наиболее часто применяется «Medical outcomes study 36-item short-form health survey» (SF-36) (Ware and Sherbourne, 1992), широко использующийся, в том числе для популяционных исследований, в различных странах мира. Среди специальных анкет широко применяется Миннесотский опросник «Minnesota living with heart failure questionnaire» (MLHFQ), разработанный в 1987 г. Т. Rector и J. Cohn, для больных с хронической сердечной недостаточностью, обладающий достаточной валидностью и высокой чувствительностью и позволяющий определить, насколько имеющаяся сердечная недостаточность ограничивает физические возможности больного, социально-экономические аспекты и общественные связи пациента, а также положительное эмоциональное восприятие жизни (Rector and Cohn, 1992). К специальным относятся также опросники, разработанные для больных артериальной гипертензией: «Описание гипертонического статуса» - «Нурегtension status inventory» (HYPER), «Опросник по оценке качества жизни при артериальной гипертензии» - «Quality of life questionnaire for arterial hypertension» (CHAL) (Soboleva and Slobodenyuk, 2013).
У больных стабильной стенокардией напряжения наиболее часто для оценки качества жизни используется «Сиэттлский опросник для больных стенокардией» - «Seattle angina questionnaire» (SAQ) (Spertus et al., 1995). Анкета SAQ состоит из 19 вопросов, оценивающих КЖ по 5 шкалам (значения от $0 \%$ до $100 \%$ ): физические ограничения, стабильность приступов стенокардии, частота приступов, удовлетворенность лечением и качество жизни (либо отношение к болезни). Выявлена достоверная корреляция между значениями каждой из шкал опросника и такими показателями как толерантность к физическим нагрузкам, тяжесть заболевания с точки зрения лечащего врача, употребление нитроглицерина. Данные об уровне КЖ, полученные с помощью SAQ, высоковоспроизводимы. Опросник обладает высокой чувствительностью к изменениям состояния пациентов с ИБС, в том числе и после оперативного лечения заболевания. Он получил широкое международное признание, переведен на 12 языков мира, применялся более чем в 20 рандомизированных исследованиях по оценке эффективности лечения больных ИБС и считается одним из наиболее адекватных опросников для определения качества жизни больных стабильной стенокардией (Dougherty et al., 1998; Zholdasbekova et al., 2016).

В последние годы не утихает интерес к омега-3 полиненасыщенным жирным кислотам ( $\omega-3$ ПНЖК) в терапии больных сердечно-сосудистыми заболеваниями в связи с выявленным множеством их позитивных эффектов у этой категории больных. Согласно литературным данным, $\omega-3$ ПНЖК обладают гиполипидемическими, антиагрегационными, антиаритмическими, противовоспалительными, вазодилатирующими и другими свойствами (Lizogub et al., 2010; Saravanan et al., 2010). Такие эффекты как гипотензивный и антиаритмический позволяют этим жирным кислотам оказывать существенное влияние на клиническое течение ИБС, тем самым улучшая качество жизни больных (Tavazzi et al., 2008). Одновременно с этим продолжаются поиски средств, способствующих дальнейшему снижению заболеваемости и смертности от сердечно-сосудистой патологии.

Следует учитывать, что больные ИБС по протокольной терапии имеют более чем достаточный набор лекарственных препаратов, что увеличивает не только стоимость лечения, но также и возможность появления побочных эффектов. С этой точки зрения особенно актуальна физиотерапия: магнитотерапия (MT), известная своими положительными эффектами на сердечно-сосудистую систему (аналгезирующий, гипотензивный, гипокоагуляционный) (Zolotarova and Petrosjan, 2004; Ulashik et al., 2015). Кроме того, необходимо подчеркнуть, что МТ отличается хорошей переносимостью, безопасным воздействием, практически не имеет противопоказаний и применяется даже у больных с подострой стадией инфаркта миокарда (Serdiuk, 2004; Zolotarova and Panigrahi, 2008). При этом следует отметить, что влияние омега-3 полиненасыщенных жирных кислот, магнитотерапии и, тем более, их сочетания на качество жизни больных ИБС в литературе освещено слабо, что диктует необходимость изучения данного вопроса.

Цель данной статьи - оптимизировать качество жизни больных стабильной стенокардией с использованием омега-3 полиненасыщенных жирных кислот и магнитотерапии. 


\section{Материал и методы исследований}

Работа выполнена на базе кардиологического отделения Военно-медицинского клинического центра Южного региона Украины, а также на базе терапевтического отделения КУ «Южненская городская больница».

В исследование включено 85 больных стенокардией напряжения I-III функциональных классов. Диагноз стабильной стенокардии установлен в соответствии с рекомендациями Ассоциации кардиологов Украины (Kovalenko et al., 2016). В исследование не включены больные с нестабильной стенокардией и инфарктом миокарда, диагностированными в течение последнего месяца, а также пациенты с серьезными нарушениями сердечного ритма, сердечной недостаточностью ІІБ - III стадии и тяжелой соматической патологией (опухоли, сепсис, кровотечения в анамнезе и др.). Все больные дали добровольное информированное согласие на участие в исследовании. Для решения поставленных задач сформировано три группы больных.

I группа (контрольная) - 28 больных. Для лечения применяли протокольный комплекс для больных стенокардией: нитраты, $\beta$-блокаторы, ингибиторы АПФ, антитромбоцитарные препараты, статины (аторвастатин в суточной дозе 20 мг).

II группа - 29 больных. На фоне протокольного комплекса применяли препарат $\omega-3$ ПНЖК (витрум кардио омега-3 (приказ МЗ Украины 27.06.06 г. № 411, p. с. UA/4642/01/01) в суточной дозировке 2000 мг, разделенных на два приема. Суточная доза аторвастатина уменьшена до 10 мг в связи с гиполипидемическим эффектом омега-3 полиненасыщенных жирных кислот.

III группа - 28 больных. К протокольному комплексу с низкотерапевтической (10 мг) дозой аторвастатина добавляли витрум кардио омега-3 и курс магнитотерапии по специальной методике: два индуктора одновременно располагали на область сердца (переменное магнитное поле, 14 мТл) и область печени (синусоидальное магнитное поле, 20 мТл). Курс лечения составлял 10 ежедневных процедур с экспозицией 20 минут, начиная с 5-7-х суток с начала медикаментозной терапии. Процедуры магнитотерапии проводили с использованием серийного аппарата ПДМТ-01 (ТУ 45-889-3293000 ТУ, № 132, 1989 г.). Переменное и синусоидальное магнитные поля выбраны как оптимальные по влиянию на сердечно-сосудистую систему (Sysoeva, 2005). Одновременное их воздействие на область сердца и область печени позволяет воздействовать на максимальный объем крови и использовать при этом минимальную индуктивность (20 и 14 мТл). Специальная методика с одновременным использованием двух видов полей с разной индуктивностью и на две зоны обуславливает теоретические предпосылки к максимальной коррекции основных этиопатогенетических звеньев ИБС (атеротромбоз, гипертензия, гиперкоагуляция, ангинозные приступы и др.), а значит и большие возможности улучшения качества жизни.

Период наблюдения составил 63,5 $\pm 3,1$ суток. Все больные до и после лечения самостоятельно заполняли опросник SAQ (значения представлены в процентах, максимальное значение соответствует наиболее высокому показателю качества жизни), а также отмечали числовое значение, соответствующее общему уровню своего самочувствия по визуальной аналоговой шкале (ВАШ), на которой наилучшее состояние здоровья отмечено как 100, а наихудшее - как 0 (Martsevich et al, 2016).

Данные обрабатывали с помощью программы Statistica 10 (StatSoft Inc., USA). Проверку соответствия данных нормальному распределению проводили с использованием критериев Колмогорова - Смирнова и Шапиро - Уилка. В случаях нормального распределения для сравнения выборок применяли ANOVA. Данные представлены как среднее значение и ошибка среднего $(\mathrm{M} \pm \mathrm{m})$. В случаях несоответствия данных нормальному распределению сравнение проводили с использованием непараметрических критериев Крускала - Уоллиса и Вилкоксона; данные представлены в виде медианы (Me) и квартилей $\left(\mathrm{Q}_{1}-\mathrm{Q}_{3}\right)$. Различия между параметрами считались достоверными при $\mathrm{P}<0,05$.

\section{Результаты}

Возраст больных колебался от 45 до 75 лет, среди них мужчин 47, женщин 38. Средний возраст больных в I, II и III группе составил соответственно $62,4 \pm 1,2,61,8 \pm 1,3$ и $63,1 \pm 1,6$ лет $(\mathrm{P}>$ $0,05)$. В I группе соотношение мужчин и женщин составило $57,1 \%$ и $42,9 \%$, во II $-55,2 \%$ и $44,8 \%$, в III - $53,6 \%$ и $46,4 \%$. Распределение больных по функциональным классам стенокардии в группах статистически не различалось ( $\mathrm{P}>0,05$, табл. 1).

\section{Таблица 1}

Распределение больных исследуемых групп по функциональным классам стенокардии

\begin{tabular}{lcccccr}
\hline \multirow{2}{*}{$\begin{array}{c}\text { Функцио- } \\
\text { нальные } \\
\text { классы } \\
\text { стенокардии }\end{array}$} & \begin{tabular}{c} 
I группа, $\mathrm{n}=28$ \\
\cline { 2 - 7 }
\end{tabular} & $\begin{array}{c}\text { число } \\
\text { больных }\end{array}$ & $\%$ & $\begin{array}{c}\text { число } \\
\text { больных }\end{array}$ & $\%$ & \multicolumn{2}{c}{$\begin{array}{c}\text { число } \\
\text { больных }\end{array}$} & $\%$ \\
\hline I & 1 & 3,6 & 2 & 6,9 & 1 & 3,6 \\
II & 13 & 46,4 & 13 & 44,8 & 9 & 32,1 \\
III & 14 & 50,0 & 14 & 48,3 & 18 & 64,3 \\
\hline
\end{tabular}

Большинство больных имели сопутствующую артериальную гипертензию, причем количество пациентов с ее наличием во всех исследуемых группах не различалось ( $89,3 \%$ в I, 86,2\% во II и $89,3 \%$ в III группе). Количество больных с хронической сердечной недостаточностью I-IIА стадии также не различалось во всех группах (92,9\% в I, 89,7\% во II и $89,3 \%$ в III группе). Таким образом, по возрасту, полу, тяжести стабильной стенокардии, а также частоте встречаемости сопутствующих заболеваний и осложнений, группы были сопоставимы между собой. Достоверных отличий между исходными показателями качества жизни у больных разных групп не отмечено ( $\mathrm{P}>0,05$, рис. 1$)$.

Также из диаграммы видно, что согласно данным опросника SAQ изначально все показатели качества жизни у больных всех исследуемых групп до лечения были снижены относительно нормативных значений, составляющих 100\% у здоровых людей. Хуже всего больные стабильной стенокардией оценили свое качество жизни по шкале частоты приступов $(20,0 \%, 20,0 \%, 20,0 \%$ в I, II, III группах соответственно; P > $0,05)$, а также по шкале стабильности течения стенокардии $(50,0 \%, 25,0 \%$, и $25,0 \%$ в I, II и III группах соответственно; P > $0,05)$. Значительно сниженные показатели физической активности, едва превышающие 50\% отметки (52,8\%, 52,8\% и 50,0\% соответственно в I, II и III группах; P > 0,05), а также удовлетворенности лечением $(56,3 \%, 56,3 \%$ и $50,0 \%$ в I, II и III группах соответственно; P > 0,05) обусловили, на наш взгляд, низкую общую оценку больными своего качества жизни $(37,5 \%, 50,0 \%$ и $41,8 \%$ в I, II и III группах соответственно; Р > 0,05). Анализ данных по визуальной аналоговой шкале также показал низкую оценку своего состояния обследованными больными $(55,0 \%$, $55,0 \%$ и 55,0\% в I, II и III группах соответственно; Р > 0,05). В целом медианы оценок по всем шкалам Сиэттлского опросника едва превышают 55\% значения, что свидетельствует о значительном негативном влиянии стабильной стенокардии напряжения на качество жизни больных.

Анализ результатов исследования качества жизни у больных I (контрольной) группы после лечения показал значительный и достоверный прирост показателей по большинству шкал SAQ на фоне терапии протокольным для стабильной стенокардии медикаментозным комплексом (рис. 2).

Несмотря на достаточно выраженное улучшение показателей стабильности стенокардии (прирост составил $34,8 \%$; $\mathrm{P}=$ $1,8 \cdot 10^{-5}$ ) и частоты стенокардии (прирост составил $11,8 \% ; \mathrm{P}=$ $\left.8,9 \cdot 10^{-5}\right)$, по шкалам ограничения физической активности, удовлетворенности лечением и визуальной оценке самочувствия прирост не был столь значительным $(+4,2 \%,+12,7 \%$ и $+11,2 \%$ соответственно; $\mathrm{P}=8,5 \cdot 10^{-5} ; 4,0 \cdot 10^{-5}$ и 0,03$)$. 


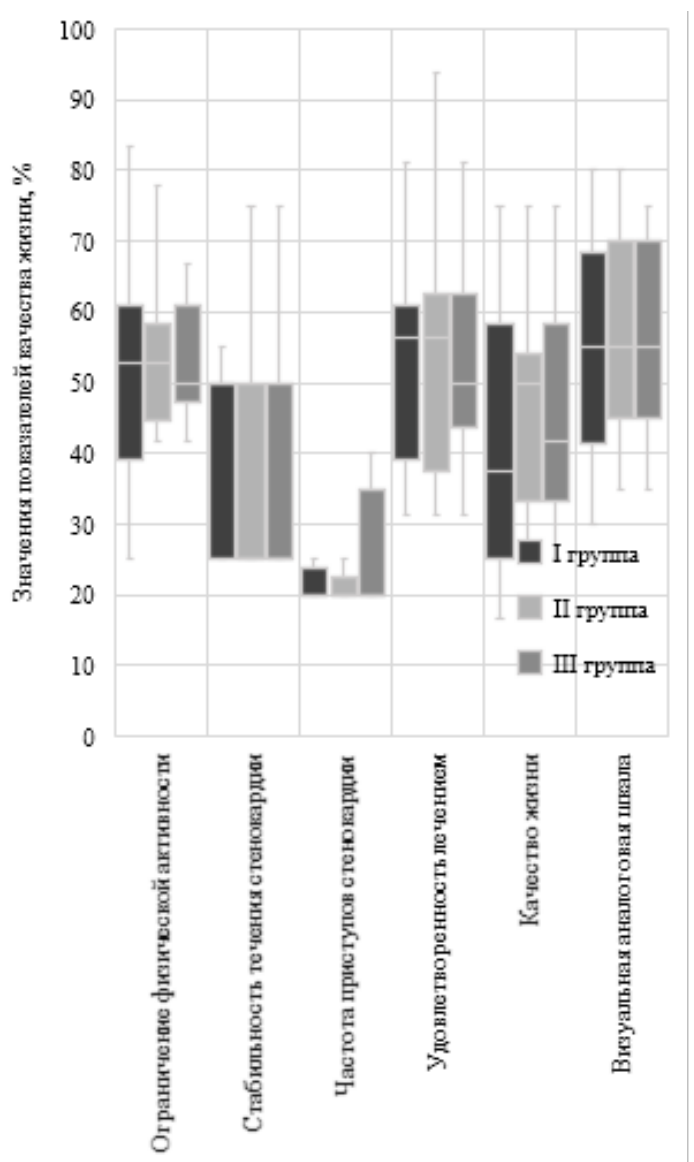

Рис. 1. Показатели качества жизни у больных исследуемых групп до лечения $\left(\mathrm{n}_{1}=28, \mathrm{n}_{2}=29, \mathrm{n}_{3}=28\right)$ :

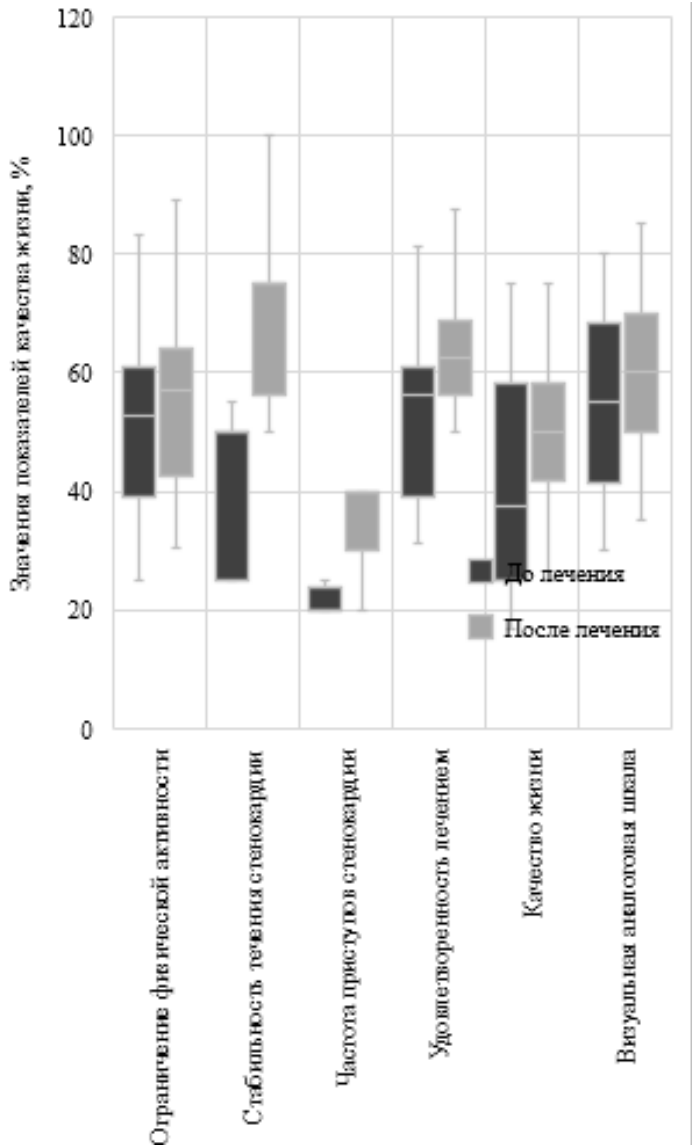

Рис. 2. Показатели качества жизни у больных I (контрольной) группы до и после лечения ( $\mathrm{n}=28)$ :

верхняя и нижняя границы прямоугольников соответствуют значениям первого и третьего квартилей, горизонтальная линия внутри прямоугольника - медиане, вертикальные линии - максимальным и минимальным значениям выборки

На наш взгляд, это обусловлено эмоциональной составляющей, которая, со слов большинства больных, сводится к осторожности и боязни увеличения своей физической активности из-за уменьшившихся, но все еще сохраняющихся приступов стенокардии. В связи с этим прирост по шкале качества жизни составил в данной группе лишь 7,2\% (с 37,5\% до 50,0\%) и был единственным из изучаемых показателей, не достигшим достоверных изменений после терапии $(\mathrm{P}=0,054)$. Результаты исследования качества жизни у больных II группы, принимавших на фоне протокольной терапии препарат омега-3 полиненасыщенных жирных кислот, показали, что после лечения все показатели SAQ и BAШ увеличились достоверно (рис. 3).

Ни один из представленных показателей своих нормативных значений не достиг. Наибольший прирост отмечен по показателям шкал стабильности течения стенокардии и частоты приступов, увеличившиеся на $41,4 \%$ и $14,5 \%$ (P $=8,3 \cdot 10^{-6}$ и $4,0 \cdot 10^{-5}$ соответственно). Также на $16,4 \%$ увеличился уровень удовлетворенности больных проводимым лечением ( $\mathrm{P}=3,6 \cdot$ $10^{-5}$ ). Намного меньше увеличились показатели шкал ограничения физической активности (с $52,8 \%$ до $55,5 \% ; \mathrm{P}=5,6 \cdot 10^{-6}$ ) и качества жизни (с 50,0 (33,3-50,0)\% до 50,0 (50,0-66,8)\%, P = $9,2 \cdot 10^{-3}$ ), прирост по которым составил $6,9 \%$ и $8,6 \%$ соответственно. Общая оценка самочувствия также изменилась достоверно, увеличившись на $15,7 \%$. Необходимо отметить, что некоторое несоответствие степени увеличения показателей физической активности и качества жизни более выраженному уменьшению симптомов стенокардии может объясняться, как уже было указано выше, отсутствием психологической адаптации больного к возможности расширения своей физической нагрузки. Больные III группы на фоне протокольного медикаментозного комплекса получали омега-3 полиненасыщенные жирные кислоты в суточной дозе 2 г и 10 -дневный курс магни- тотерапии с использованием переменного магнитного поля с величиной магнитной индукции 14 мТл на область сердца и синусоидального магнитного поля с величиной магнитной индукции 20 мТл на область печени. Изучение качества жизни у больных после лечения показало наибольший достоверный прирост показателей по всем шкалам Сиэттлского опросника, а также по данным визуальной аналоговой шкалы (рис. 4).

Практически все показатели (за исключением шкалы частоты приступов) после лечения приблизились, достигли либо превысили $60 \%$ значения. Как и в предыдущих группах, наиболее значительно возрос (на 54,6\%) показатель стабильности течения стенокардии, медиана которого достигла $100 \%\left(\mathrm{P}=5,6 \cdot 10^{-6}\right)$. Это свидетельствует о регистрации болей в груди, стеснении за грудиной или приступах стенокардии значительно реже, чем до лечения либо пациенты вообще не отмечали болей за исследуемый период. Шкалы частоты приступов и удовлетворенности лечением показали примерно равнозначный прирост $(21,1 \%$ и $24,1 \%, \mathrm{P}=5,6 \cdot$ $10^{-6}$ и 5,6 $10^{-6}$, соответственно). Это показывает, что многие больные, по мере уменьшения выраженности симптомов стенокардии и снижения необходимости в короткодействующих нитратах, отмечают меньшую обременительность приема препаратов (то есть большинство больных на вопрос анкеты касательно обременительности приема лекарств ответили: «слегка обременительно» либо «совсем не обременительно»), а также в большей степени считают, что делается все возможное для улучшения их состояния. Показатель шкалы ограничения физической активности возpoc на $12,1 \%\left(\mathrm{P}=5,6 \cdot 10^{-6}\right)$, что больше, чем в предыдущих группах, но также несколько не соответствует степени значительного уменьшения симптомов стенокардии. Увеличение по шкале качества жизни составило $19,1 \%\left(\mathrm{P}=5,6 \cdot 10^{-6}\right)$, а по визуальной шкале оценки самочувствия $-35,8 \%,\left(\mathrm{P}=6,0 \cdot 10^{-5}\right)$. Эти данные свидетельствуют о том, что, несмотря на некоторую осторожность и бо- 
язнь увеличить свою физическую активность при уменышившихся по частоте, но все еще сохраняющихся приступах стенокардии, у пациентов появляется осознание улучшения своего самочувствия и качества жизни.

Анализ качества жизни в группе больных с использованием омега-3 полиненасыщенных жирных кислот показал несколько больший прирост всех показателей по сравнению с таковыми в контрольной группе (рис. 2, 3). Однако из таблицы 2 видно, что достоверной межгрупповой разницы после лечения не отмечено $\left(\mathrm{P}_{1-2}>0,05\right)$. При этом все показатели продемонстрировали однонаправленную тенденцию к улучшению по сравнению с контролем, включая показатель качества жизни,

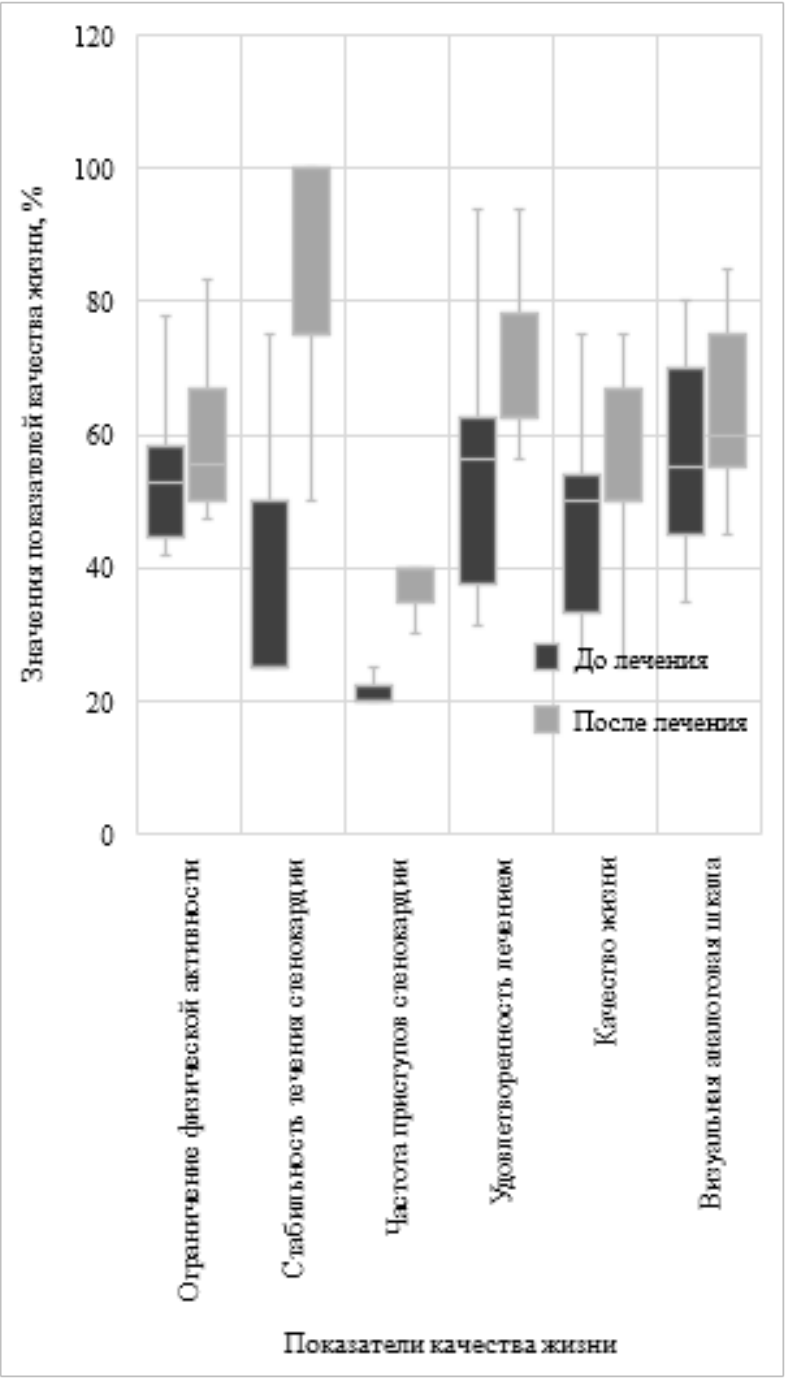

Рис. 3. Показатели качества жизни у больных II группы до и после лечения $(\mathrm{n}=29)$ : обозначения см. рис. 1 увеличившийся в контроле на $7,2 \%,(\mathrm{P}=0,054$; рис. 2$)$, а в группе с использованием $\omega$-3 ПНЖК - достоверно на 8,6\% (P = $9,2 \cdot 10^{-3}$; рис. 3$)$, однако межгрупповая разница по нему была также недостоверной $\left(\mathrm{P}_{1-2}=0,25\right)$. Это свидетельствует о способности омега-3 полиненасыщенных жирных кислот несколько улучшать качество жизни больных стабильной стенокардией не столько за счет антиангинального действия, сколько за счет своих известных эффектов по ослаблению выраженности гипертензии, сердечной недостаточности и аритмии. В итоге добавление их в протокольный комплекс, по нашим данным, значительного влияния на качество жизни больных стабильной стенокардией не оказывало.

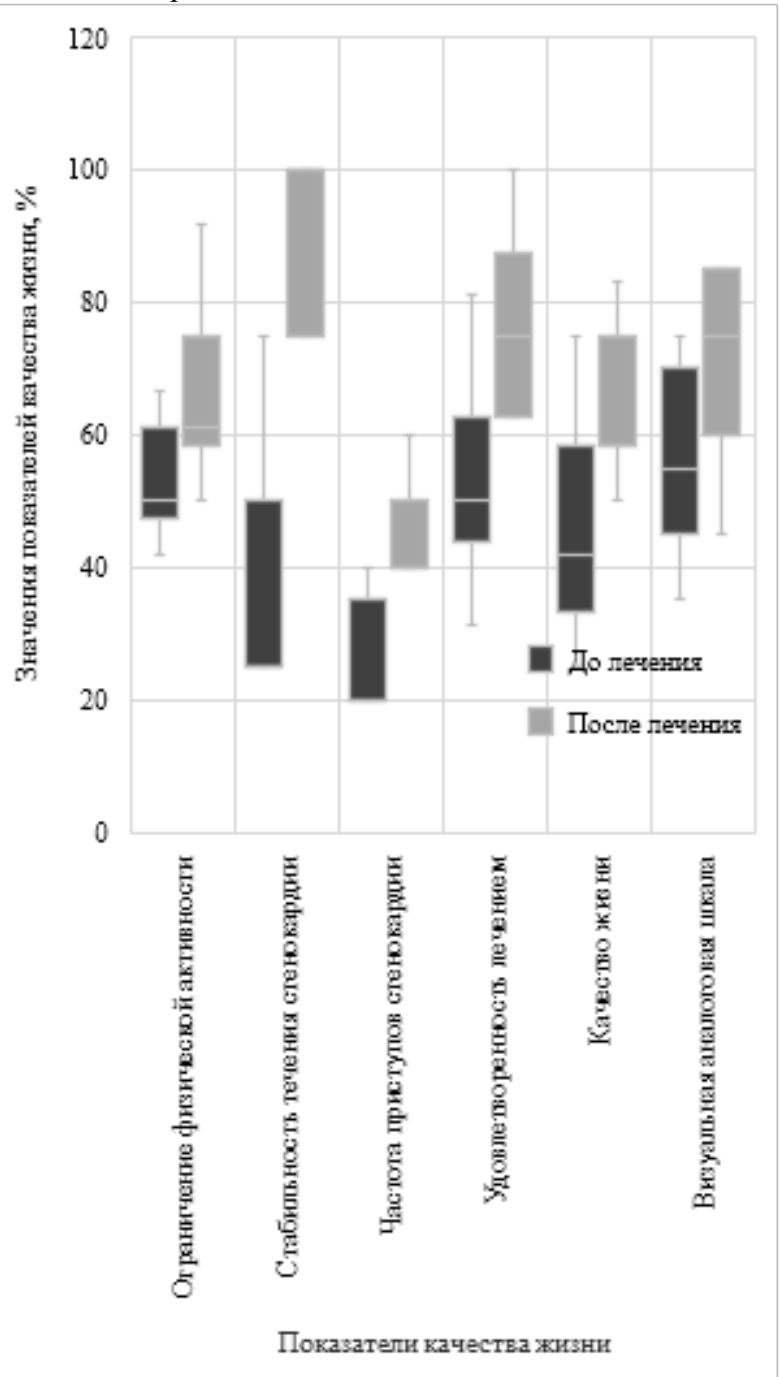

Рис. 4. Показатели качества жизни у больных III группы до и после лечения $(\mathrm{n}=28)$ : обозначения см. рис. 1

Таблица 2

Влияние терапевтических комплексов на качество жизни больных стабильной стенокардией $\left(\mathrm{Me}\left(\mathrm{Q}_{1}-\mathrm{Q}_{3}\right) \mathrm{n}_{1}=28, \mathrm{n}_{2}=29, \mathrm{n}_{3}=28\right)$

\begin{tabular}{|c|c|c|c|c|c|c|c|c|c|}
\hline \multirow{2}{*}{ Показатель } & \multicolumn{2}{|c|}{ I группа (контрольная) } & \multicolumn{2}{|c|}{ II группа ( } & \multicolumn{2}{|c|}{ 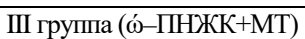 } & \multirow[b]{2}{*}{$P_{1-2}$} & \multirow[b]{2}{*}{$\mathrm{P}_{2-3}$} & \multirow[b]{2}{*}{$P_{1-3}$} \\
\hline & $\mathrm{Me}$ & $\mathrm{Q}_{1}-\mathrm{Q}_{3}$ & $\mathrm{Me}$ & $\mathrm{Q}_{1}-\mathrm{Q}_{3}$ & $\mathrm{Me}$ & $\mathrm{Q}_{1}-\mathrm{Q}_{3}$ & & & \\
\hline Шкала ограничения физической активности, \% & 56,9 & $43,13-64,0$ & 55,5 & $50,0-66,8$ & 61,0 & $58,3-75,0$ & 0,92 & 0,11 & 0,006 \\
\hline Шкала стабильности течения стенокардии, \% & 75,0 & $75,0-75,0$ & 75,0 & $75,0-100,0$ & 100,0 & $100,0-100,0$ & 0,39 & 0,03 & 0,0002 \\
\hline Шкала частоты приступов стенокардии, \% & 40,0 & $30,0-40,0$ & 40,0 & $40,0-40,0$ & 40,0 & $40,0-50,0$ & 0,70 & 0,01 & 0,0002 \\
\hline Шкала удовлетворенности лечением, \% & 62,5 & $56,3-68,8$ & 62,5 & $62,5-75,0$ & 75,0 & $62,5-87,5$ & 0,32 & 0,14 & 0,001 \\
\hline Шкала качества жизни, \% & 50,0 & $41,8-58,3$ & 50,0 & $50,0-66,8$ & 58,3 & $58,3-75,0$ & 0,25 & 0,04 & 0,0001 \\
\hline Визуальная аналоговая шкала, абс. & 60,0 & $50,0-70,0$ & 60,0 & $55,0-75,0$ & 75,0 & $60,0-85,0$ & 0,62 & 0,15 & 0,004 \\
\hline
\end{tabular}

При совместном использовании омега-3 полинасыщенных жирных кислот и магнитотерапии отмечалась достоверная межгрупповая разница с контролем по всем исследуемым показателям $\left(\mathrm{P}_{1-3}=0,006\right.$ и ниже - табл. 2). Показатель качества жизни увеличился на 19,1\%, в то время как в группе с использованием омега-3 полиненасыщенных жирных кислот - на 8,6\%, а в контрольной на 7,2\% $\left(\mathrm{P}_{1-3}=1,0 \cdot 10^{-4}\right)$. При этом следует отметить, что влияние магнитотерапии на улучшение показателей было значительно большим, чем у омега-3 полиненасышенных жирных кислот, что подтверждается достоверной межгрупповой разницей по трем из 
шести изучаемых показателей (стабильность течения стенокардии, частота приступов и качество жизни $-\mathrm{P}_{2-3}=0,03,0,01$ и 0,04 соответственно) и тенденцией, приближающейся к выраженной, у оставшихся трех $\left(\mathrm{P}_{2-3}=0,11,0,14,0,15\right.$, табл. 2$)$.

\section{Обсуждение}

Общеизвестно, что ишемическая болезнь сердца является в настоящее время главной причиной смертности в Европе, несмотря на большие усилия, направленные на лечение данной патологии (Müller-Nordhorn et al., 2008). Основными современными стратегиями терапии хронических форм ИБС является снижение риска развития сердечно-сосудистых осложнений и улучшение качества жизни больных.

В данном исследовании при изучении качества жизни больных стабильной стенокардией по опроснику SAQ и BAШ выявлено значительное снижение показателей по всем анализируемым шкалам, медианы которых едва превышали 55\%. Это свидетельствует о выраженном негативном влиянии стабильной стенокардии напряжения на качество жизни больных, что согласуется с данными Sizova et al. (2016) и является прогностически неблагоприятным, поскольку ассоциировано с повышением риска смертности и риска возникновения острого коронарного синдрома (Spertus et al., 2002). Визуальная аналоговая шкала, на наш взгляд, является несколько упрощенным и менее объективным способом оценки качества жизни, хотя некоторые авторы оценивают ее как интегральный показатель, в частности, при использовании совместно с опросником SAQ (Martsevich et al., 2016). Анализ данных по ВАШ подтвердил низкую оценку своего состояния обследованными больными (55,0\% в каждой из групп) и также совпадает с мнением других авторов (Tendera et al., 2016).

Современная стандартная медикаментозная терапия стабильной стенокардии способна, по некоторым данным, значительно уменьшить проявления болезни, однако это не всегда влечет за собой достаточное улучшение качества жизни (Marcevich et al., 2016; Tendera et al., 2016). Аналогичные результы получены в нашем исследовании. Представленное достоверное улучшение всех изучаемых показателей качества жизни у больных с использованием протокольной терапии являлось вполне ожидаемым, прежде всего, в связи с наличием в ее составе антиангинальных средств, таких как $\beta$-блокаторы и нитраты, способных значительно уменьшать частоту и ослаблять интенсивность ангинозных приступов. При этом значительного увеличения физической активности и удовлетворенности лечением не наблюдали, что обусловлено эмоциональной составляющей, которая, со слов большинства больных, сводится к осторожности и боязни увеличения своей физической активности из-за уменьшившихся, но все еще сохраняющихся приступов стенокардии. В связи с этим прирост по шкале качества жизни составил в данной группе лишь 7,2\% и был единственным из всех изучаемых показателей, не достигшим достоверных изменений после терапии $(\mathrm{P}=0,054)$.

Изучение качества жизни у больных, получавших на фоне протокольного медикаментозного комплекса омега-3 полиненасыщенные жирные кислоты, показало достоверные изменения после лечения по всем шкалам Сиэттлского опросника и визуальной аналоговой шкалы. Отмечалось некоторое несоответствие степени увеличения показателей физической активности и качества жизни при более выраженном уменьшении симптомов стенокардии, что может объясняться отсутствием психологической адаптации больного к возможности расширения своей физической нагрузки. Направленность изменений в данной группе была сходна с контролем, однако прирост показателей более выражен, включая и показатель качества жизни (рис. 2, 3), увеличившийся достоверно на 8,6\% (P=9,2 • $10^{-3}$; рис. 3), тогда как в контроле его прирост составил 7,2\%, не достигнув уровня значимости ( $\mathrm{P}=0,054$; рис. 2$)$. Несмотря на это, достоверной разницы по показателям после лечения между группами не отмечено $\left(\mathrm{P}_{1-2}>0,05\right)$, что свидетельствует об отсутствии значительного влияния омега-3 полиненасыщенных жирных кислот на качество жизни больных стабильной стенокардией. Наряду с общеизвестными данными о гиполипидемическом, антигипертензивном, антиаритмическом свойствах омега-3 ПНЖК (Saravanan et al., 2010; Lizogub et al., 2010; De Caterina, 2011), существуют немногочисленные сообщения о наличии у них антиангинального действия, заключавшегося в уменьшении количества ишемических эпизодов, по данным холтеровского мониторирования (Mihin and Mal'tseva, 2013), a также способности улучшать физическую толерантность больных ишемической болезнью сердца согласно тесту 6-минутной ходьбы (Sin'kova et al., 2014). По нашим данным, достоверного уменьшения ангинозных приступов на фоне приема омега-3 полиненасыщенных кислот не отмечено, хотя наблюдалась общая положительная тенденция по показателям данной и других шкал $(\mathrm{P}>0,05)$. Подобные результаты, возможно, обусловлены различиями в исходной характеристике больных, а также гораздо большим периодом приема препарата.

Магнитотерапия является одним из самых эффективных и безвредных физиотерапевтических факторов (Serdiuk, 2004; Zolotarova and Panigrahi, 2008), причем оптимальным воздействием на сердечно-сосудистую систему обладают, в первую очередь, переменное и синусоидальное магнитные поля (аналгезирующий, гипотензивный, антикоагулянтный и другие эффекты) (Sysoeva, 2005). Описанная выше методика позволяет их одновременное использование, причем с разной индуктивностью (14 и 20 мТл), что дает возможность оптимизации всех перечисленных эффектов и, соответственно, возможного улучшения качества жизни. Необходимо учитывать и другие известные свойства магнитотерапии: седативное действие, повышение синтеза эндорфинов, ослабление эмоционального напряжения, а также усиление под его воздействием эффектов медикаментозных средств (Ulashik, 2001; Sysoeva, 2005). Они также могут позитивно влиять на качество жизни не только при сердечно-сосудистых, но и при других заболеваниях. В нашем исследовании при совместном использовании омега-3 полинасыщенных жирных кислот и магнитотерапии эффект воздействия на все показатели качества жизни был максимальным, что подтверждается наибольшим достоверным приростом в данной группе после лечения (рис. 4). Подобное значительное увеличение исследуемых показателей свидетельствует о том, что большинство больных данной группы испытывали ангинозные приступы значительно реже, чем до лечения либо вообще не испытывали их за последние четыре недели. Помимо этого, больные ощущали значительно меньшие трудности в связи с необходимостью приема лекарственных препаратов и в меньшей степени задумывались о возможности возникновения у них инфаркта миокарда или внезапной смерти, что свидетельствует об уменьшении эмоциональной напряженности под воздействием данного медикаментозно-физиотерапевтического комплекса. Разница с контрольной группой по всем показателям достоверна $\left(\mathrm{P}_{1-3}=0,006\right.$ и ниже - табл. 2), а при анализе с группой $\omega-3$ достоверность отмечена по трем из шести показателей (стабильность течения стенокардии, частота приступов и качество жизни: $\mathrm{P}_{2-3}=0,03,0,01$ и 0,04 соответственно). На наш взгляд, это обусловлено большим влиянием магнитотерапии на ангинозные приступы на фоне приблизительно равнозначных с $\dot{\omega}-3$ ПНЖК эффектов по снижению гипертензии и выраженности хронической сердечной недостаточности. Следует учитывать также седативное действие магнитотерапии и ее эффекты по снижению эмоционального напряжения (Sysoeva, 2005), что как раз является необходимым для больных с боязнью увеличить свою физическую активность, несмотря на уменьшившуюся частоту приступов. Таким образом, кроме дополнительного анальгетического эффекта, магнитотерапия способна положительно влиять на показатели физической активности и восприятия болезни, что в итоге улучшает качество жизни больных стенокардией напряжения. 
Немаловажную роль играет потенцирующее действие магниитотерапии, способной усиливать эффекты медикаментозных средств (Ulashik, 2001), возможно, в том числе и омега-3 полиненасыщенных жирных кислот, что может служить предметом дальнейших исследований.

\section{Выводы}

Представленные данные свидетельствуют о значительном негативном влиянии стабильной стенокардии напряжения на качество жизни больных и оценку ими своего самочувствия. При использовании протокольного медикаментозного комплекса все показатели качества жизни улучшились достоверно, однако по шкалам ангинозных болей значительно, а по шкалам физической активности, оценки самочувствия и восприятия болезни - менее выраженно. При присоединении к терапии омега-3 полиненасыщенных жирных кислот отмечена слабая тенденция к улучшению показателей качества жизни, достоверно не отличающаяся от контроля. Применение комбинированной терапии с включением одновременно омега-3 полиненасыщенных жирных кислот и магнитотерапии позволило значительно и достоверно улучшить качество жизни, причем не только по шкалам, отражающим болевой компонент, но также и по показателям, которые характеризуют психологический вклад в качество жизни (удовлетворенность лечением и качество жизни (восприятие болезни)). Значительное улучшение показателей качества жизни при использовании магнитотерапии на фоне отсутствия таких влияний при самостоятельном использовании омега-3 полиненасыщенных жирных кислот обусловлено большим антиангинальным влиянием магнитотерапии, ее седативным и снимающим эмоциональное напряжение действием, а также способностью усиливать эффекты медикаментозных средств.

\section{References}

De Caterina, R. (2011). N-3 fatty acids in cardiovascular diseases. The New England Journal of Medicine, 364(9), 2439-2450.

Dougherty, C. M., Dewhurst, T., Nichol, W. P., \& Spertus, J. (1998). Comparison of three quality of life instruments in stable angina pectoris. Journal of Clinical Epidemiology, 51(7), 569-575.

Kovalenko, V. M., Lutai, M. Y., Syrenko, I. M., \& Sychov, O. S. (2016). Sertsevo-sudynni zakhvoriuvannia. Klasyfikatsiia, standarty diagnostyky ta likuvannia [Cardiovascular diseases. Classification, standards of diagnosis and treatment]. Morion, Kyiv (in Ukrainian).

Lizohub, V. H., Zavalska, T. V., Horna, O. O., Pliskevych, D. A., \& Savchenko, O. V. (2010). Metabolizm polinenasychenykh zhymykh kyslot ta yoho znachennia dlia orhanizmu liudyny [Metabolism of polyunsaturated fatty acids and its significance for the human body]. Likarska Sprava, 7-8, 21-28 (in Ukrainian)

Marcevich, S. Y., Kutishenko, N. P., \& Deev, A. D. (2016). Izuchenie vlijanija nikorandila na kachestvo zhizni patsientov so stabil'noj stenokardiej v ramkah issledovanija "KVAZAR" [The assessment of Nicorandil effect on the quality of life in patients with stable angina in the "KVAZAR" study]. Racional'naja Farmakoterapija v Kardiologii, 12(6), 654-660 (in Russian).

Mihin, V. P., \& Mal'ceva, L. S. (2013). Vlijanie omakora na variabel'nost' serdechnogo ritma i ishemicheskie izmenenija v miokarde u bol'nyh hronicheskoj ishemicheskoj bolezn'ju serdca v sochetanii s saharnym diabetom 2 tipa. [Influence of omacor on of heart rate variability and ischemic changes in the myocardium in patients with chronic ischemic heart disease in conjunction with diabetes mellitus type 2]. Arhiv Vnutrennej Mediciny, 9, 25-32 (in Russian).

Müller-Nordhom, J., Binting, S., Roll, S., \& Willich, S. N. (2008). An update on regional variation in cardiovascular mortality within Europe. European Heart Journal, 29(10), 1316-1326.

Osipov, D. A., Rozhdestvenskaja, T. V., Krom, I. L., \& Rebrov, A. P. (2011). Sootnoshenie prediktorov prognoza kachestva zhizni bol'nyh ishemicheskoj bolezn'ju serdca [Correlation of predictors of life quality prognosis of patients with coronary heart disease]. Saratovskij nauchno-medicinskij zhurnal, 7(2), 426-429 (in Russian).
Rector, T., \& Cohn, J. (1992). Assessment of patient outcome with the Minnesota living with heart failure questionnaire: Reliability and validity during a randomized, double-mind, placebo-controlled trial of pimobendan. American Heart Journal, 124(4), 1017-1025.

Saravanan, P., Davidson, N. C., Schmidt, E. B., \& Calder, P. C. (2010). Cardiovascular effects of marine omega-3 fatty acids. The Lancet, 376(9740), 540-550.

Serdiuk, V. V. (2004). Magnitoterapiya - proshloe, nystoiashchee, budushchee [Magnetotherapy - past, present, future]. Azymut - Ukraina, Kyiv (in Russian).

Sin’kova, M. N., Pepelyaeva, T. V., Isakov, L. K., Tarasov, N. I., \& Teplyakov, A. T. (2014). Otdalennye effekty vlijanija omega-3 polinenasyshhennyh zhirnyh kislot na techenie ishemicheskoj bolezni serdca u bol'nyh, perenesshih infarkt miokarda s podjemom segmenta ST na fone mnogososudistogo stenozirujushhego ateroskleroza koronarnyh arterij [Long-term effects of omega-3 polyunsaturated fatty acids on the course of ischemic heart disease in patients after ST-elevation myocardial infarction at the background of multiple-vessel lesion coronary atherosclerosis]. Kardiovaskulyarnaja Terapija i Profilaktika, 13(6), 32-37 (in Russian).

Sizova, Z. M., Zaharova, V. L., Kozlova, N. V., \& Kuchkina, T. S. (2016). Vlijanie aktivatora kalievyh kanalov nikorandila na kachestvo zhizni bol'nyh ishemicheskoj bolezn'ju serdca so stabil'noj stenokardiej naprjazhenija [Influence of the potassium channels activator nicorandil to the quality of life in patients with ishemic heart disease and stable angina pectoris]. Kardiologija, 56(6), 26-31 (in Russian).

Soboleva, M. S., \& Slobodenjuk, E. V. (2013). Ocenka vlijanija priverzhennosti k lecheniju, kachestva zhizni i zatrat pacientov na effektivnost' antigipertenzivnoj terapii [Estimating effects of medical treatment adherence, life quality and patients' expenditures for efficiency of antihypertensive therapy]. Tihookeanskij Medicinskij Zhurnal, 2, 9-13 (in Russian).

Spertus, J. A., Jones, P., McDonell, M., Fan, V., \& Fihn, S. (2002). Health status predicts long-term outcome in outpatients with coronary disease. Circulation, 106(1), 43-49.

Spertus, J. A., Winder, J. A., Dewhurst, T. A., Deyo, R. A., Prodzinski, J., McDonnell, M., \& Fihn, S. D. (1995). Development and evaluation of the Seattle Angina Questionnaire: A new functional status measure for coronary artery disease. Journal of the American College of Cardiology, 25(2), 333-341.

Sysoeva, I. V. (2005). Sovremennoe predstavlenie o biologicheskom dejstvii magnitnyh polej $\mathrm{i}$ ih primenenie $\mathrm{v}$ medicine [Modern understanding of the biological effect of magnetic fields and their application in medicine]. Medicinskie Novosti, 4, 21-28 (in Russian).

Tavazzi, L., Maggioni, A. P., \& Marchioli, R. (2008). Effect of n-3 polyunsaturated fatty acids in patients with chronic heart failure (the GISSI-HF trial): A randomized, double-blind, placebo-controlled trial. The Lancet, 372(9645), 1223-1230.

Tendera, M., Chassany, O., Ferrari, R., Ford, I., Steg, P. G., Tardif, J. C., \& Fox, K. (2016). Quality of life with ivabradine in patients with angina pectoris. Circulation: Cardiovascular Quality and Outcomes, 9, 31-38.

Ulashik, V. S. (2001). Sovremennye napravlenija razvitija magnitoterapii [Modem directions of the development of the magnetotherapy]. Vestnik Fizioterapii i Kurortologii, 7(3), 9-15 (in Russian).

Ulashik, V. S., Pletnev, A. S., Vojchenko, N. V., \& Pletnev, S. V. (2015). Magnitoterapija: Teoreticheskie osnovy i prakticheskoe primenenie [Magnetotherapy: Theoretical basis and practical application]. Belarusskaja nauka, Minsk (in Russian).

Ware, J. E., \& Sherbourne, C. D. (1992). The MOS 36-item short form health survey (SF-36): Conceptual framework and item selection. Medical Care, 30, 473-483.

Zholdasbekova, A. S., Kalmatayeva, Z. A., Nurbakyt, A. N., \& Absatarova, K. S. (2016). Izuchenie kachestva zhizni pacientov ishemicheskoj bolezn'ju serdca, perenesshih operacii po revaskuliarizacii miokarda [The study of quality of life in patients with coronary artery disease after operations of myocardial revascularization]. Vestnik Kazahskogo Nacional'nogo Medicinskogo Universiteta, 1, 654-656 (in Russian).

Zolotarova, N. A., \& Panigrahi, P. K. (2008). Sostojanie sistemy gemostaza u bol'nyh posle trombolizisa i kursa geparinoterapii i metody ego korrekcii s ispol'zovaniem magnitoterapii [The state of the hemostatic system in patients after thrombolysis and the course of heparin therapy and methods of its correction with the use of magnetotherapy]. Ukrainskyi Terapevtychnyi Zhurnal, 2, 37-41 (in Russian).

Zolotarova, N. A., \& Petrosjan, A. P. (2004). Biologicheskie effekty razlichnyh vidov magnitnyh polej i ih ispol'zovanie v lechenii IBS i degenerativnyh zabolevanij sustavov [Biological effects of different types of magnetic fields and their use in the treatment of IHD and degenerative joint diseases]. Medicinskaja Reabilitacia, Kurortologija i Fizioterapija, 39, 41-43 (in Russian). 